\title{
Serum immune markers as triggers of reactional episodes in multibacillary patients with leprosy
}

\author{
Jamile Leão Rêgo ${ }^{\text {b,d }}$, Nadja de Lima Santana ${ }^{b, d}$, \\ Eric Roberto Aguiare ${ }^{\mathrm{e}}$, Ivonete Santos Queiroz ${ }^{\mathrm{b}}$, \\ Lucas Pedreira de Carvalho ${ }^{\mathrm{a}, \mathrm{b}, \mathrm{c}, \mathrm{d}}$, Paulo Roberto Lima Machado ${ }^{\mathrm{a}, \mathrm{b}, \mathrm{d}}$ \\ \& Léa Cristina Castelluccia,b,d \\ anstituto Nacional de Ciência e Tecnologia em Doenças Tropicais, Brasil; \\ ORCID: https://orcid.org/0000-0002-9625-2469 \\ ${ }^{\mathrm{b}}$ Serviço de Imunologia, Universidade Federal da Bahia, Salvador, BA, \\ Brasil \\ ${ }^{\mathrm{c}}$ Laboratório de Pesquisa Clínica (LAPEC) do Instituto Gonçalo Moniz- \\ Fiocruz-Ba, Salvador, Bahia, Brasil \\ d Programa de Pós-graduação em Ciências da Saúde, Faculdade de \\ Medicina, Universidade Federal da Bahia, Salvador, BA, Brasil \\ ${ }^{\mathrm{e}}$ Departamento de Ciências Biológicas, Universidade Estadual de Santa \\ Cruz, Ilhéus, BA, Brasil
}

Submitted and accepted 6 October 2020

\begin{abstract}
Summary
Introduction: The development of leprosy reactions is one of the main challenges in the management of patients with leprosy. The reactions can be divided into type 1 (RR) and type 2 (ENL), representing comorbidities that can cause nerve damage and disability. This study aimed to correlate the production of immunological markers in the serum of leprosy patients with and without reactions.

Methods: Cytokines and chemokines were measured in serum by sandwich ELISA in a cross-section of 69 leprosy patients: 24 without evidence of reactions (NR), 23 with RR and 22 with ENL.

Results: We identified an increase in CXCL-9 and CXCL-10 in the multibacillary forms (MB) of leprosy that might have a role in the development of reactions, since both chemokines were positively correlated with the pro-inflammatory cytokine IL-6 in reactional patients. IL- 8 was also noted as a marker for reactions and was correlated with TNF and CCL4 in this group. Even in patients without reactions there was a strong correlation between IL- 8 and IL- 6 , which led us to think that the concomitant increase in these two markers in MB patients may signal impending reactions.
\end{abstract}

Correspondence to: Léa Cristina Castellucci, Rua João das Botas, S/N ${ }^{\circ}$, Hospital Universitário Professor Edgard Santos, Serviço de Imunologia. Salvador, Bahia, 40.110-160, Brazil. Tel.: (071) 3283-8114/8116 (e-mail: leacastel@hotmail.com) 
Conclusions: All together, we suggest CXCL-9, CXCL-10, IL-8 and CCL4 as markers for leprosy reaction development, in addition to the interactions with inflammatory cytokines incriminated in MB, such as IL-6 and TNF.

Keywords: Leprosy, leprosy reactions, cytokines, chemokines, serum

\section{Introduction}

Leprosy is a chronic infectious disease transmitted by person to person contact between someone infected with Mycobacterium leprae and another susceptible individual. ${ }^{1}$ Although its prevalence has globally decreased from $>5$ million cases in the mid-1980s to $<200000$ following introduction of multidrug therapy (MDT), this neglected disease is still a relevant public health problem in several countries, especially Brazil, India and Indonesia, which account for almost $84 \%$ of new leprosy cases globally. ${ }^{2}$ Leprosy is a complex disease, characterized by clinical forms with contrasting pathological and immunological aspects. Its spectrum comprises two opposite forms, the tuberculoid pole (TT), in which subjects develop a strong T-cell response against $M$. leprae accompanied of few lesions with a negative bacillary index; and the lepromatous pole (LL), characterized by specific T-cell anergy, multiple lesions and a high bacillary load. Besides these two poles, most subjects present the unstable borderline forms with a broad range of clinical manifestations. ${ }^{3,4}$ For diagnosis and treatment purposes, these patients can still be classified as multibacillary (MB) and paucibacillary (PB) according to WHO. During the chronic course of disease, acute inflammatory episodes, called leprosy reactions, may occur before, during or after treatment. Reactions require prolonged treatment with various drugs, including corticosteroids, thalidomide and immunosuppressive agents, but still are the main cause of deformities and disabilities. The type 1 reaction or reversal reaction ( $R R$ ) is associated with sudden inflammation of the skin lesions and/or peripheral nerves mainly in borderline subjects due to a strong cellular immune and inflammatory response against $M$. leprae antigens. ${ }^{5}$ The type 2 reaction or erythema nodosum leprosum (ENL), on the other hand, affects LL and borderline lepromatous (BL) patients and cause painful subcutaneous nodules as well as systemic symptoms such as fever, myalgia, asthenia, arthritis, and vasculitis. Immunologically, ENL reflects the deposition of immune complexes and also a pro-inflammatory cytokine production. ${ }^{6,7}$ It is generally accepted that the various forms of leprosy are determined essentially by the host response to M. leprae. Many studies point to the role of cytokines and chemokines in all clinical forms of the disease. . $^{3,8,11}$ Cytokines are glycoproteins produced by a wide variety of stimuli either by immune or non-immune cells, orchestrating the immune system. Defects in cytokine production or their expression at the target tissue sites are associated with pathologic states. ${ }^{12}$ Chemokines are potent chemoattractors of specific leucocyte subsets, thus leading to the variable cellular infiltration of leprosy lesions. ${ }^{13}$ The CXC group, which contains IL-8, predominantly attracts neutrophils whereas the CC group, which contains CCL2 (MCP-1), CCL3 (MIP-1 $\alpha$ ) and CCL4 (MIP-1 $\beta$ ), predominantly attracts monocytes and lymphocytes. In the current literature, there are few studies evaluating serum levels of cytokines in leprosy patients using the enzymelinked immunosorbent assay (ELISA) technique, with some controversial results in the various forms of leprosy. ${ }^{12}$ The aim of this study was to evaluate the production of immunological markers in the serum of leprosy patients with and without leprosy reactions, in order to validate these molecules as biomarkers of disease. 


\section{Methods}

\section{SUBJECTS AND BLOOD COLLECTION}

Using the cross-sectional model, sixty-nine leprosy cases of both sexes, aged between 18 to 65 years, were selected from two leprosy outpatient reference centers in the city of Salvador, Brazil: Hospital Universitário Professor Edgard Santos and Hospital Couto Maia. Patients were diagnosed according to the Brazilian's Ministry of Health guidelines, which provides a protocol for classification on the Ridley-Jopling spectrum and by the WHO field classification, ${ }^{3,14}$ as previously reported by others. ${ }^{15-17}$ Participants were classified into three groups: patients with leprosy per se and without reactions (HAN group), patients with type 1 reactions (RR group) or with type 2 reactions (ENL group). All patients read and signed the informed consent form and were treated according to WHO/MDT. Patients who were on therapy with steroids, thalidomide or having any other systemic illness were excluded from the study. Blood $(8 \mathrm{~mL})$ was collected into Vacutainers ${ }^{\circledR}$ tubes without anticoagulant (Becton Dickinson) and centrifuged at $20000 \mathrm{~g}$ for ten minutes. Hemolyzed samples were discarded. The serum obtained was stored at $-70^{\circ} \mathrm{C}$ until use.

\section{ELISA CYTOKINE ASSAYS}

Levels of the cytokines IL-1 $\beta$, IL-6, IFN- $\gamma$ and IL-12 p40, IL-17, and IL-10 were measured in serum using commercial kits from R\&D (R\&D systems Inc. Minneapolis, MN, USA) and BD OptEIA $^{\text {TM }}$ Set human (BD Biosciences, San Jose, CA, USA), respectively, according to the manufacturers' protocols. To measure the TNF levels, a high sensitivity ELISA sandwich technique was used $\left(\mathrm{NOVEX}^{\circledR}\right.$, Thermo Fisher, Inc), also following the manufacturer's instructions. Optical density was measured in the spectrophotometer at $450 \mathrm{~nm}$. The results were expressed in $\mathrm{pg} / \mathrm{ml}$, based on comparisons with standard curves for each cytokine kit.

\section{ELISA CHEMOKINE ASSAYS}

Levels of the chemokines IL-8, CCL3, CCL4 and CCL2, CXCL-9, CXCL-10 were measured in serum using commercial kits from R\&D (R\&D systems Inc. Minneapolis, MN, USA) and BD OptEIA ${ }^{\mathrm{TM}}$ Set human (BD Biosciences, San Jose, CA, USA), respectively, according to the manufacturers' protocols. Optical density was measured in the spectrophotometer at $450 \mathrm{~nm}$. The results were expressed in $\mathrm{pg} / \mathrm{ml}$, based on comparisons with standard curves for each chemokine kit.

\section{STATISTICAL ANALYSIS}

Correlations in cytokine production were assessed through Pearson correlation coefficient, $r$ (confidence interval $>95 \%$ ) where significance ( $p$-value) was indicated as follow: “*” for $p<0.05$, “**” for $p<0.01$ and "***" for $p<0.001$. Differences in cytokine and chemokine abundance between groups were assessed through non-parametric two-sided Mann-Whitney test for comparison of two independent groups. Only statistically significant differences were indicated on the plot. The plots were generated in $\mathrm{R}$ using corrplot and ggplot 2 packages.

\section{Results}

CHARACTERISTICS OF THE PATIENTS WITH LEPROSY

Detailed characteristics of subjects are shown in Table 1 . The majority were female over forty years old. Forty-six patients were classified as MB (BB, BL and LL) and twenty three as PB (U, TT, BT and Neural). The mean ages were $43.04 \pm 12.97$ and $50.43 \pm 13.99$ 
Table 1. Clinical and demographic characteristics, and bacillary index of leprosy subjects from Salvador, Bahia, Brazil

\begin{tabular}{|c|c|c|c|c|}
\hline Patients with leprosy & $\begin{array}{c}\text { No reaction - } \\
\text { Controls }(N=24)\end{array}$ & $\begin{array}{c}\text { Leprosy reaction - Cases } \\
\text { RR }+\operatorname{ENL}(N=45)\end{array}$ & $\begin{array}{c}\text { Type } 1 \text { reaction } \\
\operatorname{RR}(N=23)\end{array}$ & $\begin{array}{l}\text { Type } 2 \text { reaction } \\
\text { ENL }(N=22)\end{array}$ \\
\hline Age $($ mean $\pm \mathrm{SD})$ & $45.21(15.45)$ & $45.67( \pm 12.80)$ & $49.47(14.59)$ & $41.68(9.38)$ \\
\hline \multicolumn{5}{|l|}{ Gender } \\
\hline Female & $15(62.5)$ & $22(48.9)$ & $15(65.2)$ & $8(36.4)$ \\
\hline Male & $9(37.5)$ & $23(51.1)$ & $8(34.8)$ & $14(63.6)$ \\
\hline \multicolumn{5}{|l|}{$\begin{array}{l}\text { Ridley-Jopling } \\
\text { classification }\end{array}$} \\
\hline TT & $5(20.8)$ & - & - & - \\
\hline $\mathrm{BT}$ & $4(16.6)$ & $8(17.8)$ & $8(34.8)$ & - \\
\hline $\mathrm{BB}$ & $3(12.5)$ & $8(17.8)$ & $7(30.4)$ & $1(4.5)$ \\
\hline $\mathrm{BL}$ & $2(8.3)$ & $6(13.3)$ & $3(13)$ & $3(13.6)$ \\
\hline LL & $5(20.8)$ & $22(48.9)$ & $4(17.4)$ & $18(81.6)$ \\
\hline I & $5(20.8)$ & - & - & - \\
\hline Other (Neural) & - & $1(2.2)$ & $1(4.3)$ & - \\
\hline Total & 24 & 45 & & \\
\hline \multicolumn{5}{|c|}{ Bacillary index $*(N=54)$} \\
\hline & 0.69 & 2.07 & 0.62 & 3.3 \\
\hline
\end{tabular}

$\mathrm{SD}$ = standard deviation; I, Indeterminate; BT, borderline tuberculoid; BB, borderline borderline; BL, borderline lepromatous; LL, lepromatous leprosy, RR, Reversal Reaction; ENL, Erythema Nodosum Leprosum.

*The mean bacillary load presented by each study group is described represented by a logarithmic scale between 0 and $6+$.

years, respectively. A total of fifty-four individuals presented the initial bacillary index (BI) at diagnosis. Of these, 21 patients had a negative initial BI, while all others had positive smear microscopy greater than $0.1+$. Fifteen did not present BI on admission $(22 \%)$, because some were referred only for reaction treatment or were admitted with the initial tests already performed and their classification defined; even so, confirmatory analyses were performed for the disease and its forms.

CYTOKINE AND CHEMOKINE PROFILES IN LEPROSY PATIENTS ACCORDING TO THE PRESENCE OR ABSENCE OF REACTIONS

Using the ELISA technique we looked at circulating levels of cytokines and chemokines in serum of leprosy patients with reactions (combining RR + ENL, thereby called REAC) and without reactions (HAN). Among the cytokines, patients from the REAC group produced significantly higher levels of IL-6 $(2.7 \pm 6.02 \mathrm{pg} / \mathrm{ml}$ vs $0.35 \pm 0.84)$, TNF $(2.3 \pm 1.97 \mathrm{pg} / \mathrm{ml}$ vs $1.60 \pm 1.55)$ and $\mathrm{IL}-10(11.6 \pm 34.6 \mathrm{pg} / \mathrm{ml}$ vs $3.2 \pm 3.63)$ as compared to the HAN group. Regarding the chemokines evaluated, we observed that patients with reactions produced significantly more IL-8 $(105.36 \pm 172.75 \mathrm{pg} / \mathrm{ml}$ vs $54.91 \pm 122.93 \mathrm{pg} / \mathrm{ml})$ and CCL4 (108.47 $\pm 129.82 \mathrm{pg} / \mathrm{ml}$ vs $55.55 \pm 91.09 \mathrm{pg} / \mathrm{ml}$ ) as compared to patients without reactions, as shown in Figure 1. No differences were observed for the other cytokines and chemokines evaluated. Likewise, there were no significant associations comparing RR vs ENL patients $(p>0.05$, data not shown).

\section{CYTOKINE AND CHEMOKINE PROFILES BETWEEN MB VERSUS PB PATIENTS}

We stratified our analysis by comparing within the HAN group (i.e., subjects free of reactions), the multibacillary (MB) versus paucibacillary (PB) patients in order to identify immunological markers that could divide the groups. Corroborating previous data, IL-6 and IL-10 levels were 


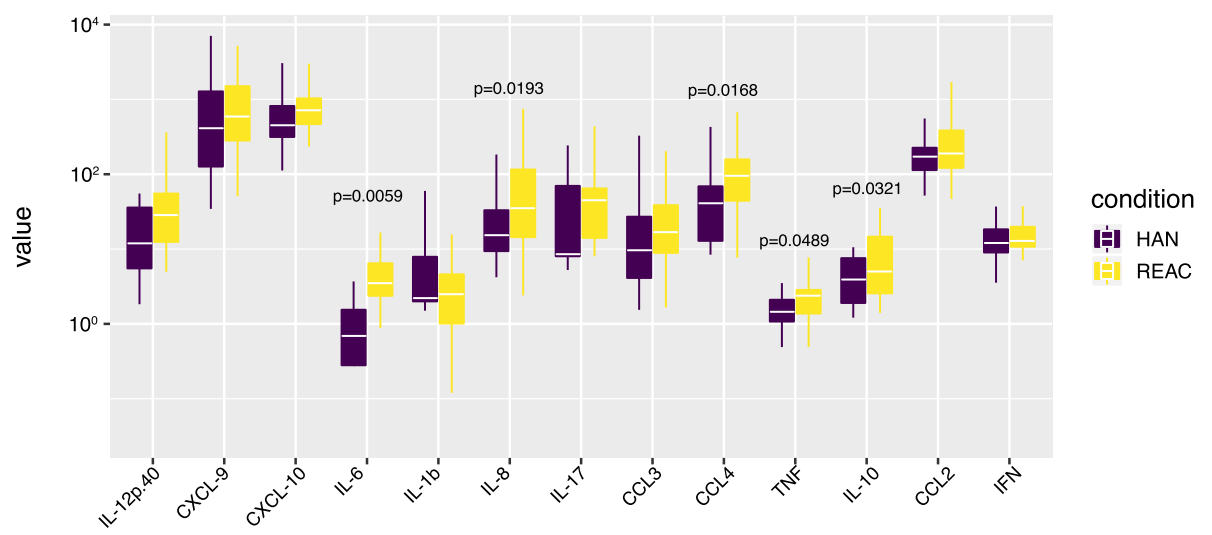

Figure 1 Differential production of cytokines and chemokines between HAN and REAC groups. Box plot of cytokine production grouped by non-reactional patients (HAN) $v s$ patients with reactions (REAC). Statistical significance was assessed using Mann-Whitney test and significant $p$-values are indicated.

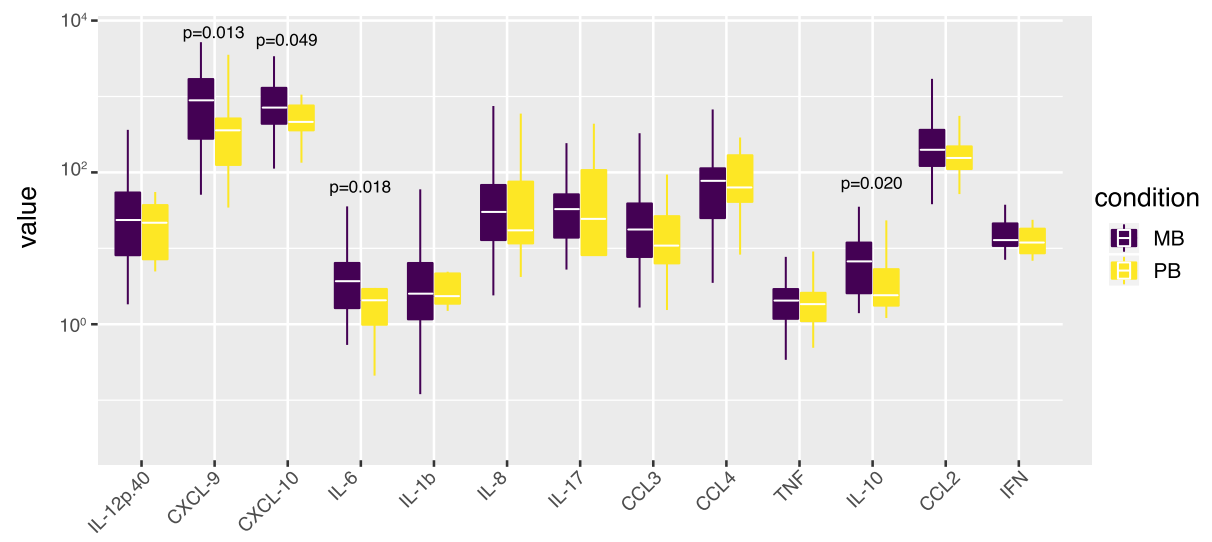

Figure 2 Differential production of cytokines and chemokines between PB and MB groups. Box plot of cytokine production grouped by paucibacillary (PB) and multibacillary (MB). Statistical significance was assessed using Mann-Whitney test and significant $p$-values are indicated.

higher and associated with MB $(0.61 \pm 1.15 \mathrm{pg} / \mathrm{ml}$ vs $0.12 \pm 0.36 \mathrm{pg} / \mathrm{ml}$ and $4.29 \pm 4.32 \mathrm{pg} / \mathrm{ml}$ vs $2.47 \pm 2.99 \mathrm{pg} / \mathrm{ml}$, respectively) as shown in Figure 2. In addition, the chemokines CXCL-9 $(1222.3 \pm 946.91 \mathrm{pg} / \mathrm{ml}$ vs $926.99 \pm 1965.81 \mathrm{pg} / \mathrm{ml})$ and CXCL-10 $(903.46 \pm 1036.43 \mathrm{pg} / \mathrm{ml}$ vs $670.56 \pm 592.39 \mathrm{pg} / \mathrm{ml}$ ) were also raised as markers for the MB phenotype, Figure 2.

\section{CORRELATION ANALYSIS FOR PATIENTS WITH LEPROSY PER SE AND LEPROSY REACTIONS}

The Pearson correlation coefficient (r) was applied over the serum levels of chemokines and cytokines within the HAN group. We found positive correlations between IL-10 and both, IL-1 $\beta$ and CXCL-9. It is also remarkable that correlations between CCL4 and IL-17 with CXCL-10, INF- $\gamma$ and IL-8. IL-8 were in turn, strongly correlated with IL-6. All these correlations are shown in Figure 3. We then applied the r coefficient in patients from the REAC group. Correlation was observed between the triad IL-8, TNF and CCL4. Equally, there was a 


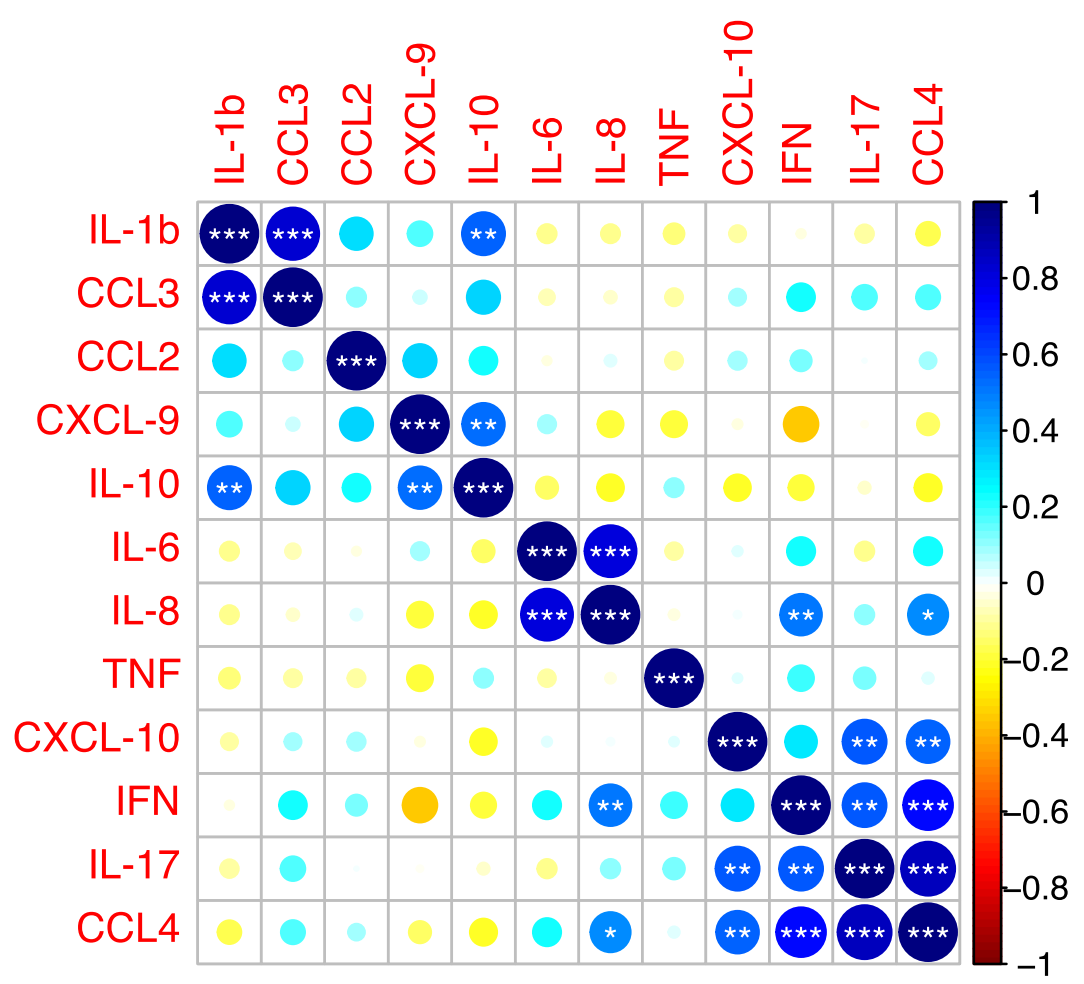

Figure 3 Analysis of correlation among cytokines and chemokines in leprosy patients without reactions (HAN). Red and blue dots indicate negative and positive Pearson correlation, respectively. $p$-value is indicated as follow: "*” for $p<0.05$, “**” for $p<0.01$ and “***" for $p<0.001$. Non-significant correlation $p$-values were omitted.

correlation between IL-6 and the chemokines CXCL-9 and CXCL-10 in that group, as shown in Figure 4.

\section{Discussion}

Leprosy reactions are the main cause of disabilities and remain a major challenge in leprosy control, requiring long-term treatment with drugs associated with several side effects. In this sense, several studies have been carried out with the aim of finding relevant biomarkers for the diagnosis or prognosis of reactions. For example, CXCL-10 and IL-6 have been shown to be potential plasma markers for the diagnosis of RR whereas IL-7, granulocyte colony stimulating factor (G-CSF) and IL-6 were proposed as a profile related to ENL. ${ }^{7}$ In the current study, we bring two main contributions: first, we corroborated in serum the role of cytokines and chemokines previously associated with leprosy pathogenesis in different models; second, we confirmed a leading role for CCL4 and IL-8 as serological biomarkers of leprosy reactions. In a previous study, CCL3, CCL4 and IL-8 genes were down modulated at both THP1 cells and nerve biopsies infected by $M$. leprae, suggesting that the inhibition of chemokines by $M$. leprae would be a possible survival strategy for the pathogen. ${ }^{18}$ In this context, we believe that increased levels of these chemokines in the serum of patients with leprosy reactions indicate the host's attempt to process tissue and circulating bacillary antigens and quell infection, while, 


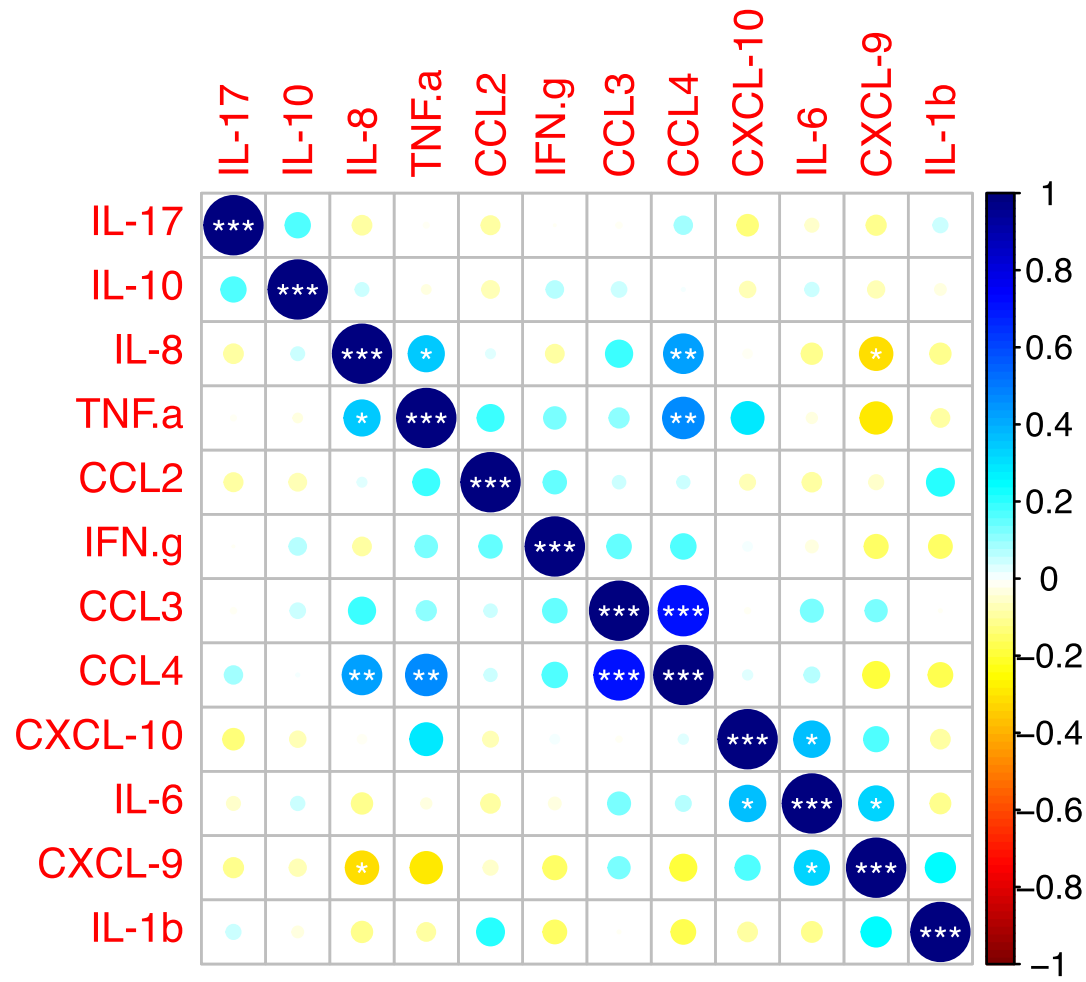

Figure 4 Analysis of correlation among cytokines and chemokines in patients with reactions (REAC). Red and blue dots indicate negative and positive Pearson correlation, respectively. $p$-value is indicated as follow: “*” for $p<0.05$, “**” for $p<0.01$ and “***” for $p<0.001$. Non-significant correlation $p$-values were omitted.

on the other hand, exacerbating the inflammation contributing to the establishment of the reaction. Furthermore, our data is also in agreement with a previous multifamily genetic scan that identified the 17q21 region, enriched in chemokine genes such as CCL2, CCL3, CCL4 as a locus linked to leprosy. ${ }^{19}$ IL- 8 allows for the activation and chemotaxis of neutrophils toward the inflamed tissues. TNF has been shown to induce IL-8 secretion in whole blood, the majority of which is derived from monocytes. In addition, data from cell lineage shows that TNF is a potent and rapid inducer of IL- 8 protein secretion and gene expression after NF- $\kappa \beta$ binding in the IL- 8 promoter region..$^{20,21}$ IL- 8 was found in skin biopsies from lesions across the leprosy spectrum and also in reactional patients. ${ }^{13,22,23}$ Furthermore, there are data showing that LL leprosy monocytes spontaneously expressed high levels of IL-8 as compared to controls. ${ }^{24}$ Our data shows that this chemokine can also be a relevant serological marker for leprosy reactions due to its higher production as compared with patients without reaction. In addition, confirming previous data, both IL-6 and IL-10 levels were lower in HAN subjects as compared to REAC patients. IL- 6 was previously seen as the only biomarker of type 1 and 2 reactions and higher levels of IL- 6 were associated with both MB disease and the occurrence of type 2 reactions. ${ }^{6,7,25,26}$ Concerning IL-10, studies have shown higher expression of IL-6, IL-8, and IL-10 mRNA in lesions of ENL patients as well as higher IL-10 serum levels in MB and ENL patients. ${ }^{23,27}$ 
Regarding our correlation analysis, we highlight a positive correlation between the chemokines CCL4 and IL-8 themselves, and also with TNF in REAC patients. Concerning the CCL3-CCL4 correlation, considering that these two chemokines are located in the same chromosomal region we might think that upon $M$. leprae challenge a common regulatory mechanism activates their pathway bringing them up together. Concomitantly, other immunological events such as the production of IL- 8 and co-regulated cytokines such as TNF, IL-6 and IL-10 are activated. Still, regarding the REAC group, we found a correlation between the production of IL-6 with CXCL-9 and CXCL-10. These three molecules were also significantly higher within the MB group of patients, which reinforce them as potential prognostic markers for leprosy reactions. Serum samples revealed elevation of circulating CXCL-10 associated with RR episodes, suggesting this chemokine as a marker that differentiates between RR and borderline forms of disease. ${ }^{28,29}$ Regarding CXCL-9, we found very little data in the literature, but there is a report showing raised levels after type I reactions. ${ }^{30}$

Considering the HAN group, we found a correlation between IL-10 with IL-1 $\beta$ and CXCL9. This correlation could be influenced by the MB patients. In this case, it is possible to postulate that IL-10 begins to rise in the multibacillary forms of the disease in an attempt to modulate the pro-inflammatory activity of these molecules, and then remains elevated after the development of reactions. Additionally, we found a correlation between CCL4 and IL17 with INF- $\gamma$, CXCL-10 and IL-8, as well as between IL-6 and IL-8 in this group. IL-17 synergizes with IFN- $\gamma$ to enhance the production of pro-inflammatory cytokines by human keratinocytes, including IL-6 and IL-8, thereby increasing the migration of T cells to the skin. ${ }^{31}$ It is reasonable that the higher production of IFN- $\gamma$ in these patients favors individuals who did not develop reactions, contributing to a more effective cellular immunity against the bacilli.

The serum production of cytokines and chemokines mirrors inflammatory processes and might serve as disease markers of different stages and clinical forms. Unfortunately, ELISAs may vary due to detection methods, distinct sensitivities as well as interferences caused by drugs and possible associated pathologies. Based on these limitations it is important to recognize that the role of immunological molecules in serum needs further clarification. Nevertheless, they are still useful as markers of inflammation and allow us to correlate their production pattern with immunopathological aspects of the disease. We highlight a consistent production of chemokines CCL4, IL-8, CXCL-9 and CXCL-10 in the serum of patients with reactions, as well as a correlation that indicates that these chemokines rise in parallel with important pro-inflammatory cytokines such as IL-6 and TNF as well as the regulatory IL-10 in $\mathrm{MB}$ patients.

\section{Review board approval}

Approval for the use of the samples in this study was obtained from the Ethical Committee of the Hospital Universitário Prof. Edgard Santos, Federal University of Bahia (number 891.963).

\section{Conflict of interest}

The authors report no conflict of interest.

\section{Funding}

This work was funding by the National Institute of Science and Technology in Tropical Diseases, Brazil (N 573839/2008-5). 


\section{Authors' contribution}

JLR, NLS and ISQ collected samples and carried out the ELISAs in the laboratory; ERA performed the statistical analysis using R; PRLM participated in the collection of clinical data and revised the manuscript; LPC revised the manuscript; LCC supervised the lab work and wrote the manuscript. All authors read and approved the final version of this article.

\section{Acknowledgments}

We thank the staff of leprosy clinics from Hospital Universitário Professor Edgard Santos and Hospital Couto Maia for support in the recruitment of subjects and sample collection.

\section{References}

1 Lastória JC, de Abreu MAMM. Leprosy: review of the epidemiological, clinical, and etiopathogenic aspects Part 1. Anais Brasileiros De Dermatologia, 2014; 89(2): 205-218.

2 WHO. Global leprosy update, 2017: reducing the disease burden due to leprosy. Weekly Epidemiol Rec, 2018; 93(35): 445-456.

3 Ridley D, Jopling W. Classification of leprosy according to immunity. A five-group system. Int J Lepr Other Mycobact Dis, 1966; 34(3): 255-273.

4 Ridley DS, Jopling WH. A classification of leprosy for research purposes. Lepr Rev, 1962; 33: 119-128.

5 Scollard DM, Smith T, Bhoopat L, Theetranont C, Rangdaeng S, Morens DM. Epidemiologic characteristics of leprosy reactions. Int J Lepr Other Mycobact Dis, 1994; 62(4): 559-567.

6 Moraes MO, Sarno EN, Almeida AS, Saraiva BC, Nery JA, Martins RC et al. Cytokine mRNA expression in leprosy: a possible role for interferon-gamma and interleukin-12 in reactions (RR and ENL). Scand J Immunol, 1999; 50: 541-549, doi:10.1046/j.1365-3083.1999.00622.x.

7 Stefani MM, Guerra JG, Sousa ALM, Costa MB, Oliveira MLW, Martelli CT et al. Potential plasma markers of type 1 and type 2 leprosy reactions: a preliminary report. BMC Infect Dis, 2009; 9(1): 1-8.

8 Ottenhoff THM. Immunology of leprosy: lessons from and for leprosy. Int J Lepr Other Mycobact Dis, 1994; 62(1): 108-121.

9 Sampaio EP, Sarno EN. Expression and cytokine secretion in the states of immune reactivation in leprosy. Braz Med Biol Res, 1998; 31: 69-76.

10 Stefani MMA, Martelli CMT, Gillis TP, Krahenbuhl JL. Brazilian Leprosy Study G. In situ type 1 cytokine gene expression and mechanisms associated with early leprosy progression. J Infectious Dis, 2003; 188(7): 1024-1031.

11 Van Brakel WH, Khawas I, Lucas SB. Reactions in leprosy: an epidemiological study of 386 patients in west Nepal. Lepr Rev, 1994; 65(3): 190-203.

12 Kawatra Madan N, Agarwal K, Chander R. Serum cytokine profile in leprosy and its correlation with clinicohistopathological profile. Lepr Rev, 2011; 82: 371-382.

13 Kirkaldy AA, Musonda AC, Khanolkhar-Young S, Suneetha S, Lockwood DNJ. Expression of CC and CXC chemokines and chemokine receptors in human leprosy skin lesions. Clin Exp Immunol, 2003; 134(3): 447-453.

14 WHO. WHO Expert Committee on Leprosy. World Health Organ Tech Rep Ser, 1988; 768: 1-51.

15 Machado PRL, Machado LM, Shibuya M, Rego J, Johnson WD, Glesby MJ. Viral Co-infection and Leprosy Outcomes: A Cohort Study. PLoS Neglected Trop Dis, 2015; 9(8): e0003865.

16 Moura Oliveira J, Leão Rêgo J, de Lima Santana N, Braz M, Jamieson S, Silva Vieira T et al. The $-308 \mathrm{bp}$ TNF gene polymorphism influences tumor necrosis factor expression in leprosy patients in Bahia State, Brazil. Infection, Genet Evol, 2016; 39: 147-154, doi:10.1016/j.meegid.2016.01.026.

17 Rêgo JL, de Lima Santana N, Machado PRL, Ribeiro-Alves M, de Toledo-Pinto TG, Castellucci LC et al. Whole blood profiling of leprosy type 1(reversal) reactions highlights prominence of innate immune response genes. BMC Infect Dis, 2018; 18(1): 422, doi:10.1186/s12879-018-3348-6.

18 Guerreiro LTA, Robottom-Ferreira AB, Ribeiro-Alves M, Toledo-Pinto TG, Rosa Brito T, Rosa PS et al. Gene Expression Profiling Specifies Chemokine, Mitochondrial and Lipid Metabolism Signatures in Leprosy. PLoS ONE, 2013; 8(6): e64748.

19 Jamieson SE, Miller EN, Black GF, Peacock CS, Cordell HJ, Howson JMM et al. Evidence for a cluster of genes on chromosome 17q11-q21 controlling susceptibility to tuberculosis and leprosy in Brazilians. Genes Immu, 2004; 5(1): 46-57.

20 Fujishima S, Hoffman AR, Vu T, Kim KJ, Zheng H, Daniel D et al. Regulation of neutrophil interleukin 8 gene expression and protein secretion by LPS, TNF- $\alpha$, and IL-1 $\beta$. J Cell Physiol, 1993; 154(3): 478-485. 
21 Vlahopoulos S, Boldogh I, Casola A, Brasier AR. Nuclear factor- $\kappa$ B-Dependent Induction of Interleukin-8 Gene Expression by Tumor Necrosis Factor alpha: Evidence for an Antioxidant Sensitive Activating Pathway Distinct From Nuclear Translocation. Blood, 1999; 94(6): 1878-1889.

22 Moraes MO, Sarno EN, Almeida AS, Saraiva BC, Nery JA, Martins RC et al. Cytokine mRNA Expression in Leprosy: A Possible Role for Interferon- $\gamma$ and Interleukin-12 in Reactions (RR and ENL). Scand J Immunol, 1999; 50(5): 541-549.

23 Yamamura M, Wang XH, Ohmen JD, Uyemura K, Rea TH, Bloom BR et al. Cytokine patterns of immunologically mediated tissue damage. J Immunol, 1992; 149(4): 1470-1475.

24 Hasan Z, Mahmood A, Zafar S, Khan AA, Hussain R. Leprosy Patients with Lepromatous Disease Have an Up-regulated IL-8 Response That Is Unlinked to TNF- $\alpha$ Responses. Int J Lepr Other Mycobact Dis, 2004; 72: 35-44.

25 Belgaumkar VA, Gokhale NR, Mahajan PM, Bharadwaj R, Pandit DP, Deshpande S. Circulating cytokine profiles in leprosy patients. Lepr Rev, 2007; 78: 223-230.

26 Ochoa MT, Valderrama L, Ochoa A, Zea A, Escobar CE, Moreno LH et al. Lepromatous and tuberculoid leprosy: clinical presentation and cytokine responses. Int J Dermatol, 1996; 35(11): 786-790.

27 Moubasher AD, Kamel NA, Zedan H, Raheem DD. Cytokines in leprosy, I. Serum cytokine profile in leprosy. Int J Dermatol, 1998; 37: 733-740, doi:10.1046/j.1365-4362.1998.00381.x.

28 Scollard DM, Chaduvula MV, Martinez A, Fowlkes N, Nath I, Stryjewska BM et al. Increased CXC Ligand 10 Levels and Gene Expression in Type 1 Leprosy Reactions. Clin Vaccine Immunol, 2011; 18(6): 947-953.

29 Sharma R, Singh P, Loughry WJ, Lockhart JM, Inman WB, Duthie MS et al. Zoonotic Leprosy in the Southeastern United States. Emerg Infect Dis, 2015; 21(12): 2127-2134.

30 Geluk A, van Meijgaarden KE, Wilson L, Bobosha K, van der Ploeg-van Schip JJ, van den Eeden SJF et al. Longitudinal Immune Responses and Gene Expression Profiles in Type 1 Leprosy Reactions. J Clin Immunol, 2014; 34(2): 245-255.

31 Nedoszytko B, Sokołowska-Wojdyło M, Ruckemann-Dziurdzińska K, Roszkiewicz J, Nowicki RJ. Chemokines and cytokines network in the pathogenesis of the inflammatory skin diseases: atopic dermatitis, psoriasis and skin mastocytosis. Postepy Dermatol Alergol, 2014; 31(2): 84-91. 